\title{
How strongly does appetite counter weight loss? Quantification of the homeostatic control of human energy intake
}

Authors: David Polidori ${ }^{1}$; Arjun Sanghvi²; Randy Seeley ${ }^{3}$; Kevin D. Hall ${ }^{2 *}$

Affiliations: ${ }^{1}$ Janssen Research \& Development, LLC, San Diego, CA; ${ }^{2}$ National Institute of Diabetes and Digestive and Kidney Diseases, National Institutes of Health, Bethesda, MD; ${ }^{3}$ Department of Surgery, University of Michigan, Ann Arbor, MI.

*To whom correspondence should be addressed:

Kevin D. Hall, PhD

National Institute of Diabetes \& Digestive \& Kidney Diseases

National Institutes of Health

12A South Drive, Room 4007

Bethesda, MD 20892-5621

phone: 301-402-8248

fax: 301-402-0535

email: kevinh@niddk.nih.gov

Revision Date: April 29, 2016.

Word Count: 3904

Funding: This research was supported by the Intramural Research Program of the NIH, National Institute of Diabetes \& Digestive \& Kidney Diseases, using data from a study sponsored by Janssen Research \& Development, LLC.

Disclosure: D.P. is a full-time employee of Janssen Research \& Development, LLC.

K.D.H. reports patent pending on a method of personalized dynamic feedback control of body weight (US Patent Application No. 13/754,058; assigned to the NIH) and has received funding from the Nutrition Science Initiative to investigate the effects of ketogenic diets on human energy expenditure. R.S. is a paid consultant for Janssen, Novo 
Nordisk, Takeda, Daichii Sankyo, Novartis, Pfizer, Nestle, Circuit Therapeutics and

Ethicon. R.S., also has received research support from Novo Nordisk, Ethicon, Sanofi and Boehringer Ingelheim. A.S. reports no conflicts of interest. 


\section{What is already known about this subject?}

- Human body weight is believed to be regulated by homeostatic feedback control of both energy intake and energy expenditure.

- Adaptations of energy expenditure to weight loss have been well-established, but the homeostatic control of energy intake has yet to be quantified.

\section{What this study adds:}

- We provide the first quantification of the homeostatic control of energy intake in free-living humans.

- The increase in energy intake per kg of weight lost is several-fold larger than the known energy expenditure adaptations.

- Homeostatic control of energy intake is likely the primary reason why it is difficult to achieve and sustain large weight losses. 


\section{Abstract}

Objective: To quantify the homeostatic feedback control of energy intake in response to long-term covert manipulation of energy balance in free-living humans.

Methods: We used a validated mathematical method to calculate energy intake changes during a 52 week placebo-controlled trial in 153 patients treated with canagliflozin, a sodium glucose co-transporter inhibitor that increases urinary glucose excretion thereby resulting in weight loss without patients being directly aware of the energy deficit. We analyzed the relationship between the body weight time course and the calculated energy intake changes using principles from engineering control theory.

Results: We discovered that weight loss leads to a proportional homeostatic drive to increase energy intake above baseline by 100 kcal/day per kg of lost weight - an amount more than 3-fold larger than the corresponding energy expenditure adaptations.

Conclusions: While energy expenditure adaptations are often thought to be the main reason for slowing of weight loss and subsequent regain, feedback control of energy intake plays an even larger role and helps explain why long-term maintenance of a reduced body weight is so difficult. 


\section{Introduction}

Body weight is believed to be regulated by homeostatic control of both energy intake and energy expenditure. Several carefully controlled feeding experiments in humans have quantified how energy expenditure adapts in response to alterations of energy intake and body weight. For example, Leibel and colleagues found that energy expenditure changed by several hundreds of kcal/day thereby acting to resist weight changes (1). Such data have enabled the development of accurate mathematical models of energy expenditure and body weight dynamics in humans in response to given changes in energy intake (2). In contrast, energy intake adaptations have been difficult to accurately quantify in humans despite the widespread belief that homeostatic control of energy intake is critical for body weight regulation $(3,4,5)$ and acts as part of a complex neurobiological system to determine overall human food intake behavior $(6,7)$.

Why has the assessment of human homeostatic energy intake control lagged the quantification of energy expenditure changes with weight loss? First, we lacked the ability to accurately measure changes in free-living energy intake in large numbers of people over extended time periods. While accurate energy intake measurements can be performed while subjects are housed in laboratory setting, such studies are typically of short duration and the artificial nature of the environment makes it difficult to translate the results to the real world $(8,9)$. Indeed, free-living energy intake is known to fluctuate widely from day to day and exhibits little short-term correlation with energy expenditure or body weight $(10,11)$. Therefore, observations over long time scales are required to investigate regulation of human energy balance and body weight (12) making laboratory 
based studies impractical.

Unfortunately, free-living subjects are notorious for being unable to provide accurate estimates of energy intake using self-report methods (13) and the expense and difficulty of employing objective biomarker methods severely limits their applicability (14). To address this important problem, we recently developed an inexpensive mathematical method for calculating energy intake changes in free-living subjects using repeated body weight data $(15,16)$. This method was validated using data from 140 free-living people who participated in a two year calorie restriction study where the mean calculated energy intake changes at all time points were within $40 \mathrm{kcal} /$ day of measurements obtained using an expensive biomarker method (16).

The second impediment to quantifying energy intake control in humans is that we lacked an intervention that increases energy output without participants consciously knowing that this is occurring. Rather, most interventions that alter body weight or energy expenditure also evoke central responses that may mask the effect of weight changes per se on the homeostatic control of energy intake in humans. For example, engaging in an exercise program might increase energy expenditure and lead to weight loss, but exercise is a conscious behavior that doesn't have an effective placebo control. Furthermore, exercise has a complex role in modulating appetite (17) and may induce compensatory changes in other components of total energy expenditure that are difficult to quantify. Therefore, changes in energy intake during an exercise program may not solely be due to homeostatic mechanisms and are likely to also involve conscious changes in behavior. 
Here, we used data from a placebo-controlled trial in patients with type 2 diabetes who were treated for one year with canagliflozin, an inhibitor of sodium glucose transporter 2 (SGLT2), thereby increasing energy output in the form of urinary glucose excretion (UGE) (18). In patients with type 2 diabetes, treatment with canagliflozin at a dose of $300 \mathrm{mg} /$ day increases mean daily UGE by approximately $90 \mathrm{~g} /$ day which is sustained at the same level throughout the duration of treatment (19). This leads to a net energy loss of $\sim 360 \mathrm{kcal} /$ day that occurs without directly altering central pathways controlling energy intake and without the patients being directly aware of the energy deficit. In other words, SGLT2 inhibition provides a novel way to perturb human energy balance that largely bypasses the volition of the subjects. Any observed increased energy intake countering the weight loss induced by SGLT2 inhibition therefore likely reflects the activity of the homeostatic feedback control system. We calculated the free-living energy intake changes in 153 patients treated with $300 \mathrm{mg} /$ day canagliflozin over a 52 week trial using the measured body weight data and an assumed mean UGE of $90 \mathrm{~g} /$ day as inputs to a mathematical model that has recently been validated against an expensive biomarker method (16). We found that the homeostatic feedback control of energy intake in humans was proportional to the amount of weight lost and was substantially stronger than the control of energy expenditure. These findings have important implications for interpreting the results of obesity interventions.

\section{Methods}

\section{Calculating changes in energy intake during canagliflozin treatment}


We used measured body weight, BW, and baseline patient characteristics in the previously published placebo-controlled trail of canagliflozin (18) where compliance was monitored by pill counts. All of the subjects in the canagliflozin cohort who completed the study had compliance of at least $75 \%$ and all but 3 subjects in the placebo cohort who completed the study had $>75 \%$ compliance. We calculated the changes in energy intake, $\Delta E I$, for each subject using a validated mathematical method (16) using the following equation:

$$
\Delta E I_{i}=\rho \frac{d B W_{i}}{d t}+\varepsilon\left(B W_{i}-B W_{0}\right)+\frac{\Delta \delta}{(1-\beta)} B W_{0}+U G E
$$

The inputs to the model were the change of body weight versus baseline over each interval, $\left(B W_{i}-B W_{0}\right)$, and the moving average of the measured body weight time course was used to calculate the rate of change of body weight over each interval, $d B W_{i} / d t$. The interval length was $t=(N-1) * T$, where $N=2$ was the number of body weight measurements per interval and $T=52$ was the number of days between measurements. Three subjects treated canagliflozin and 2 subjects treated with placebo had missing body weight data at one time point and linear interpolation was used to impute the missing data.

Equation 1 is a linearization of a mathematical model of adult body weight dynamics that was developed and validated using data obtained primarily from controlled feeding studies in adult humans with longitudinal measurements of changes in body composition 
as well as both resting and total energy expenditure $(20,21,22)$. The model parameter $\rho$ was the effective energy density associated with the body weight change:

$$
\rho=\frac{\eta_{F M}+\rho_{F M}+\alpha \eta_{F F M}+\alpha \rho_{F F M}}{(1-\beta)(1+\alpha)}
$$

(Equation 2)

and $\varepsilon$ defined how energy expenditure depends on body weight:

$$
\varepsilon=\frac{1}{(1-\beta)}\left[\frac{\gamma_{F}+\alpha \gamma_{L}}{(1+\alpha)}+\delta_{0}+\Delta \delta\right]
$$

(Equation 3).

The model parameters $\gamma_{F F M}$ and $\gamma_{F M}$ are the regression coefficients relating resting metabolic rate to fat-free mass (FFM) and fat mass (FM), respectively. Parameters $\rho_{\mathrm{FM}}$ and $\rho_{\mathrm{FFM}}$ are the energy densities associated with changes in FM and FFM, respectively. Most physical activities involve locomotion and have an energy cost that is proportional to body weight for a given intensity and duration of activity (23). The baseline physical activity parameter was $\delta_{0}$ and $\Delta \delta$ represents changes in physical activity that can be informed by objective measurements, if available. Without such measurements, physical activity changes are often assumed to be zero $(\Delta \delta=0)$ with the realization that the calculated energy intake changes may be in error, especially at the individual level where substantial physical activity changes can occur throughout a study. Averaging over many individuals with the assumption that $\Delta \delta=0$ can also result in a biased mean energy intake change of the group, but our previous validation study demonstrated that this bias is likely to be $<40 \mathrm{kcal} /$ day when studying $>100$ individuals (16). 
The parameter $\beta$ accounts for the adaptation of energy expenditure during a diet perturbation, $\Delta E I$, and was determined using data from eight human studies that measured changes in body composition as well as both total and resting energy expenditure before and after achieving a period of long-term stability at a lower weight (21). Parameters $\eta_{F M}$ and $\eta_{F F M}$ account for the biochemical cost of tissue deposition and turnover assuming that the change of FFM is primarily accounted for by body protein and its associated water (24).

The parameter $\alpha$ represents the relationship between changes of lean and fat mass: $\alpha \equiv d F F M / d F M=C / F M$ where $\mathrm{C}=10.4 \mathrm{~kg}$ is the Forbes parameter (25). This simple model of body composition change provides more accurate predictions than more complex models (26) and has been demonstrated to be consistent across different ethnic groups and sexes (27). For modest weight changes, $\alpha$ can be considered to be approximately constant with $F M$ fixed at its initial value $\mathrm{FM}_{0}$. The larger the initial fat mass, $\mathrm{FM}_{0}$, the smaller the parameter $\alpha$, as previously described (22). The parameter $U G E$ represents the energy losses as a result of increased urinary excretion of glucose with canagliflozin treatment. Model parameter values are given in Table 1.

In addition to validating our mathematical model using body composition and energy expenditure data from controlled feeding studies $(20,21,22)$, the model has also demonstrated accurate weight loss predictions regardless of medication usage or 
comorbid conditions, including type 2 diabetes, in free-living individuals with obesity following reduced calorie diets (28).

\section{Modeling feedback control of energy intake}

While many hormonal and neuronal factors are known to be involved in the regulation of food intake $(3,5)$, general properties of the feedback relationship between changes in body weight and changes in energy intake have not been well characterized or quantified. We tested the ability of two potential feedback models to describe the observed body weight profiles in response to sustained canagliflozin treatment.

In the first model, referred to as "proportional control”, changes in the signals regulating food intake depend only on the current body weight and are not dependent on the duration or rate of weight loss. In this model, the aggregate effect of different feedback signals regulating body weight are described by the equation:

$$
\Delta E I(t)=-k_{P} \times \Delta B W(t)
$$

where the parameter $k_{P}>0$ quantifies the feedback strength. To simulate how proportional control of energy intake affects body weight kinetics during canagliflozin treatment, we added a UGE term to a validated mathematical model of adult human body weight dynamics (22) and calculated the mean $\Delta E I$ time course during placebo treatment to capture the typical transient weight loss effect of being in the trial (see Figure 1). Equation 4 was then added to the placebo energy intake to simulate the mean homeostatic 
proportional control of $\Delta E I$ during canagliflozin treatment. The best fit parameter $k_{P}$ was determined by a downhill simplex algorithm (29) implemented using Berkeley Madonna software (version 8.3; http://www.berkeleymadonna.com) to minimize the sum of squares residuals between the simulation outputs and the measured mean body weight and the calculated mean $\triangle E I$.

We also investigated another possible model for homeostatic body weight regulation that was previously suggested (30) such that changes in energy intake also depend on how long body weight has deviated from baseline, which is expressed as an integral term quantified by a parameter $k_{I}>0$ :

$$
\Delta E I(t)=-k_{P} \times \Delta B W(t)-k_{I} \int_{0}^{t} \Delta B W(\tau) d \tau
$$

(Equation 5)

The integral feedback control was simulated using Equation 5 with the best fit value of $k_{P}$ from Equation 4 and $k_{I}=1 \mathrm{kcal} / \mathrm{kg} / \mathrm{d}^{2}$.

\section{Results}

Table 2 represents the characteristics of the type 2 diabetic subjects who completed the 52 week study and had body weight measurements throughout. The full study cohort was previously reported (18). In response to the sustained increase in UGE with canagliflozin treatment, mean body weight declined and reached a new equilibrium several kilograms lower and significantly more than the placebo group whose mean body weight loss was less than $1 \mathrm{~kg}$ (Figure 1A). To explain the measured body weight changes in the treatment group given the estimated increases in UGE, energy intake was calculated to have increased by $\sim 350 \mathrm{kcal} /$ day at steady state (Figure 1B) which is similar to recent 
estimates of the mean energy intake changes during 90 weeks of empagliflozin treatment, another SGLT2 inhibitor (31). In the placebo group, mean energy intake was calculated to transiently decrease by $\sim 100 \mathrm{kcal} /$ day over the first several weeks and return to baseline after 15 weeks (Figure 1B)

Note that as body weight declined during SGLT2 inhibition energy intake increased until it compensated for the loss of calories via UGE, after which, a new equilibrium was reached. This general pattern of response, in which a sustained perturbation (caloric loss via UGE) leads to a new equilibrium at a lower body weight, suggests that the endocrine signals responding to weight loss (such as leptin) act as part of a proportional feedback control system as shown in Figure 2 illustrating that proportional feedback control (as defined by Equation 4 with the parameter $k_{P}=95 \mathrm{kcal} /$ day per $\mathrm{kg}$ ) mimics the observed body weight and energy intake patterns in humans receiving SGLT2 inhibitors.

In contrast, despite the persistent increase in UGE, if the system regulating body weight included integral feedback control (Equation 5), body weight would ultimately have been restored to baseline values even in the presence of sustained increases in UGE. This outcome is a well-known result from control theory that integral feedback is a necessary and sufficient condition for a system to produce zero steady-state error (i.e., return to baseline body weight) in response to a sustained perturbation (i.e., persistently increased UGE) and that a proportional feedback system will always have a non-zero steady-state error (i.e., a sustained reduction in body weight) in response to a sustained perturbation (32). In contrast, for any value of $k_{\mathrm{I}}>0$, a system including integral feedback in the 
regulation of energy intake would have only transient weight loss during sustained SGLT2 inhibitor treatment as shown for the integral feedback model in Figure 2.

By modeling the mean changes in energy intake obtained with SGLT2 inhibitor treatment using the proportional feedback model, our results quantify the strength of homeostatic energy intake control in humans. On average, energy intake increased by 100 kcal/day per kg of weight lost—an effect substantially greater than the $\sim 30 \mathrm{kcal} / \mathrm{kg} /$ day changes in energy expenditure observed with 10 to $20 \%$ weight loss in subjects with obesity (1).

To put our results into context, consider the body weight trajectory depicted in Figure 3A that resulted from long-term participation in a structured commercial weight loss program (33) and illustrates the ubiquitous body weight time course characterized by initial weight loss, a plateau after 6-8 months, followed by slow weight regain (34). The calculated energy intake corresponding to this mean body weight trajectory is illustrated in Figure 3B showing an initial reduction of $\sim 700 \mathrm{kcal} /$ day from baseline followed by an exponential relaxation towards baseline over the ensuing months. The estimated energy expenditure corresponding to this intervention is also shown in Figure 3B and indicates relatively minor changes in comparison to the energy intake changes. Note that at the point of maximum weight loss occurring at the $\sim 8$ month plateau, energy intake had already returned to within $100 \mathrm{kcal} /$ day of baseline. After 1 year, the average energy intake was practically at baseline levels while body weight was still reduced by $\sim 5 \mathrm{~kg}$. A similar pattern of relapsing diet adherence has been observed using objective biomarker methods during an intensive 2 year calorie restriction study where calorie intake was 
much lower during the early period of weight loss than after the body weight had plateaued $(16,35)$.

The calculated energy intake time course during the lifestyle intervention suggests that diet adherence lapsed very early with subjects returning to their previous caloric intake and a corresponding weight plateau followed by slow weight regain. While this might be interpreted as indicating that the participants rapidly decreased their effort to adhere to the intervention, it is enlightening to consider their food intake behavior within the context of the proportional feedback control system. The dashed curve in Figure 3B illustrates the energy intake pattern corresponding to the increased homeostatic drive to eat in response to the body weight changes shown in Figure 3A. The difference between the homeostatic drive to eat and the actual energy intake is depicted in Figure 3C and is a quantitative index of the ongoing effort to sustain the intervention in the face of the continuing biological signals to overeat. In this context, a substantial persistent effort is required to avoid overeating above baseline during the intervention despite the average energy intake returning to near baseline levels.

\section{Discussion}

In the absence of ongoing efforts to restrain food intake following weight loss, homeostatic feedback control of energy intake will result in eating above baseline levels with an accompanying acceleration of weight regain. Such behavior has been previously observed in rodent models when a return to ad libitum feeding following diet restriction resulted in hyperphagia until the lost weight was regained (36). This phenomenon has 
been also observed in lean men following experimental semi-starvation (37) or short-term underfeeding (38, 39, 40). Hyperphagia in these studies was believed to result from homeostatic signals arising from loss of both body fat and lean tissues (41, 42), but a conscious desire to regain lost weight cannot be ruled out and may have contributed to the increased food intake.

Previous studies of energy intake regulation in humans have employed short-term diet manipulations to measure compensatory changes in energy intake $(43,44,45,46)$. While such studies can provide useful information about the influence of episodic appetite signals on short-term modulation of energy intake, the results cannot be readily extrapolated to the long time scales associated with regulation of human energy balance and do not provide information about how weight changes influence energy intake.

Long-term inhibition of SGLT2 provides a unique probe for assessment of human energy homeostasis since the mechanism of action is clear, its effect on energy output is consistent, and the intervention is unlikely to directly affect central pathways involved in regulation of food intake. In contrast, other interventions aimed at increasing energy expenditure, such as exercise (17) or exogenous delivery of thyroid hormone (47), have pleiotropic effects and their impact on energy expenditure can be highly variable.

The suggestion that the signals controlling energy intake act as a proportional feedback system without integral feedback is consistent with the roughly proportional changes in appetite-regulating hormones that occur rapidly in response to weight loss and do not 
further increase as weight loss is sustained (as would occur with integral feedback) (48). We do not yet know whether the simple proportional controller represented by Equation 4 is valid for a range of weight losses. For example, it may be possible that small weight changes are uncompensated by changes in energy intake such that the control system engages only after sufficient weight loss to cross some threshold (49). Furthermore, larger weight losses may result in energy intake adaptations corresponding to a nonlinear function of body weight change. Future research is required to address these questions and more fully characterize the homeostatic energy intake control system, characterize its variability between individuals, and identify its physiological mediators in humans.

Proportional homeostatic control of energy intake may help explain why the calculated exponential decay of diet adherence during weight loss interventions markedly contrasts with self-reported measurements that indicate persistence of diet adherence and no significant differences in caloric consumption between the period of early weight loss compared with the later time when weight has plateaued (50). This has led to speculation that the 6-8 month weight plateau may be entirely due to slowing of metabolic rate rather than loss of diet adherence (51). Our results suggest otherwise and self-reported energy intake measurements are well-known to be quantitatively unreliable (13). Nevertheless, the relative constancy self-reported energy intake over the first 6 months corresponds well with the calculated persistent effort to resist the homeostatic drive to overeat at above baseline levels. Therefore, self-reported measurements of diet may more accurately reflect the perceived effort of the dieter to adhere to the intervention rather than their actual energy intake. 
An important limitation of our study is that all of the subjects had type 2 diabetes and it is unclear whether our results translate to people without diabetes. For example, people with type 2 diabetes have a characteristically decreased insulin secretion in the context of insulin resistance that may quantitatively affect the feedback control of energy intake (3, 4, 5). Another limitation is that we cannot be certain that weight loss achieved by mechanisms other than SGLT2 inhibition will lead to the same homeostatic response to increase energy intake. Furthermore, we restricted our analysis to the group mean rather than attempt to characterize individual responses since UGE was not directly measured in each subject but was assumed to be $\sim 90$ g/day based on previous measurements in people with type 2 diabetes treated with $300 \mathrm{mg}$ /day canagliflozin (19, 52, 53). A subset of subjects in this study underwent a meal tolerance test where UGE was measured; their mean increase in UGE over the 3h meal test was $14.7 \mathrm{~g}$ (54); this is similar to the value observed following meals in other studies where mean 24h UGE was measured to be 90 g/day and suggests that the mean daily UGE in this study is unlikely to be substantially different than what was assumed. Therefore, there is a correspondingly small uncertainty in the mean calculated proportional control of energy intake ( 100 kcal/day per $\mathrm{kg}$ of body weight lost) at the group level. Measuring daily UGE in individuals during longterm studies with SGLT2 inhibitors would enable the individual subject variability in the magnitude of the compensatory energy intake responses to be characterized.

In summary, our results provide the first quantification of homeostatic feedback control of energy intake in free-living humans. We found that appetite proportionately increased 
by $100 \mathrm{kcal} /$ day above baseline for every kilogram of lost weight. In comparison, energy expenditure adaptations to weight loss are several-fold lower in magnitude. Therefore, homeostatic feedback control of energy intake is likely a primary reason why it is so difficult to achieve large sustained weight losses in patients with obesity. Rather, weight regain is typical in the absence of heroic and vigilant efforts to maintain behavior changes in the face of an omnipresent obesogenic environment (55). Unfortunately, there is no evidence that the energy intake feedback control system resets or relaxes with prolonged maintenance of lost weight - an effect similar to the long-term persistent suppression of energy expenditure in weight-reduced humans (56). Therefore, the effort associated with a weight loss intervention persists until either body weight is fully regained or energy intake increases above baseline to match the homeostatic drive to eat. Permanently subverting or countering this feedback control system poses a major challenge for the development of effective obesity therapies.

\section{Acknowledgements}

Canagliflozin has been developed by Janssen Research \& Development, LLC, in collaboration with Mitsubishi Tanabe Pharma Corporation. We thank Marc L. Reitman and Aaron Cypess for their helpful comments on the manuscript.

DP and KDH had full access to all the data in the study and take responsibility for the integrity of the data and the accuracy of the data analysis. $\mathrm{DP}, \mathrm{KDH}$ and $\mathrm{RS}$ designed the study; AS, DP, and KDH performed the analyses; DP, KDH, and RS interpreted the data and drafted the manuscript. 
These data were previously presented, in part, in abstract form at the International

Congress on Obesity on May 2, 2016 in Vancouver, Canada and at the 50th Annual

Meeting of the European Association for the Study of Diabetes on September 15-19, 2014 in Vienna, Austria.

\section{References}

1. Leibel RL, Rosenbaum M, Hirsch J. Changes in energy expenditure resulting from altered body weight. $N$ Engl J Med 1995;332: 621-628.

2. Hall KD. Modeling metabolic adaptations and energy regulation in humans. Annu Rev Nutr 2012;32: 35-54.

3. Gautron L, Elmquist JK, Williams KW. Neural Control of Energy Balance: Translating Circuits to Therapies. Cell 2015;161: 133-145.

4. Schwartz MW, Woods SC, Porte D, Jr., Seeley RJ, Baskin DG. Central nervous system control of food intake. Nature 2000;404: 661-671.

5. Woods SC, D'Alessio DA. Central control of body weight and appetite. J Clin Endocrinol Metab 2008;93: S37-50.

6. Berthoud HR. The neurobiology of food intake in an obesogenic environment. Proc Nutr Soc 2012;71: 478-487.

7. Hall KD, Hammond RA, Rahmandad H. Dynamic interplay among homeostatic, hedonic, and cognitive feedback circuits regulating body weight. American journal of public health 2014;104: 1169-1175.

8. Gibbons C, Finlayson G, Dalton M, Caudwell P, Blundell JE. Metabolic Phenotyping Guidelines: studying eating behaviour in humans. The Journal of endocrinology 2014;222: G1-12.

9. Stubbs RJ, Johnstone AM, O'Reilly LM, Poppitt SD. Methodological issues relating to the measurement of food, energy and nutrient intake in human laboratory-based studies. Proc Nutr Soc 1998;57: 357-372. 
10. Edholm OG, Adam JM, Healy MJ, Wolff HS, Goldsmith R, Best TW. Food intake and energy expenditure of army recruits. Br J Nutr 1970;24: 1091-1107.

11. Tarasuk V, Beaton GH. Day-to-day variation in energy and nutrient intake: evidence of individuality in eating behaviour? Appetite 1992;18: 43-54.

12. Chow CC, Hall KD. Short and long-term energy intake patterns and their implications for human body weight regulation. Physiol Behav 2014;134: 60-65.

13. Schoeller DA. How accurate is self-reported dietary energy intake? Nutr Rev 1990;48: 373-379.

14. Racette SB, Das SK, Bhapkar M, Hadley EC, Roberts SB, Ravussin E, et al. Approaches for quantifying energy intake and \%calorie restriction during calorie restriction interventions in humans: the multicenter CALERIE study. Am J Physiol Endocrinol Metab 2012;302: E441-448.

15. Hall KD, Chow CC. Estimating changes in free-living energy intake and its confidence interval. Am J Clin Nutr 2011;94: 66-74.

16. Sanghvi A, Redman LA, Martin CK, Ravussin E, Hall KD. Validation of an inexpensive and accurate mathematical method to measure long-term changes in free-living energy intake. Am J Clin Nutr 2015.

17. Blundell JE, Gibbons C, Caudwell P, Finlayson G, Hopkins M. Appetite control and energy balance: impact of exercise. Obes Rev 2015;16 Suppl 1: 67-76.

18. Stenlof K, Cefalu WT, Kim KA, Jodar E, Alba M, Edwards R, et al. Long-term efficacy and safety of canagliflozin monotherapy in patients with type 2 diabetes inadequately controlled with diet and exercise: findings from the 52-week CANTATA-M study. Current medical research and opinion 2014;30: 163-175.

19. Devineni D, Polidori D. Clinical Pharmacokinetic, Pharmacodynamic, and DrugDrug Interaction Profile of Canagliflozin, a Sodium-Glucose Co-transporter 2 Inhibitor. Clinical pharmacokinetics 2015;54: 1027-1041.

20. Hall KD, Guo J, Dore M, Chow CC. The progressive increase of food waste in America and its environmental impact. PLoS One 2009;4: e7940.

21. Hall KD, Jordan PN. Modeling weight-loss maintenance to help prevent body weight regain. Am J Clin Nutr 2008;88: 1495-1503.

22. Hall KD, Sacks G, Chandramohan D, Chow CC, Wang YC, Gortmaker SL, et al. Quantification of the effect of energy imbalance on bodyweight. Lancet 2011;378: 826-837. 
23. Schoeller DA, Jefford G. Determinants of the energy costs of light activities: inferences for interpreting doubly labeled water data. Int J Obes (Lond) 2002;26: 97-101.

24. Hall KD. Mathematical modelling of energy expenditure during tissue deposition. Br J Nutr 2010;104: 4-7.

25. Forbes GB. Lean body mass-body fat interrelationships in humans. Nutr Rev 1987;45: 225-231.

26. Thomas D, Das SK, Levine JA, Martin CK, Mayer L, McDougall A, et al. New fat free mass - fat mass model for use in physiological energy balance equations. Nutr Metab (Lond) 2010; 7: 39.

27. Broyles ST, Bouchard C, Bray GA, Greenway FL, Johnson WD, Newton RL, et al. Consistency of fat mass--fat-free mass relationship across ethnicity and sex groups. Br J Nutr 2011;105: 1272-1276.

28. Brady I, Hall KD. Dispatch from the field: is mathematical modeling applicable to obesity treatment in the real world? Obesity (Silver Spring) 2014;22: 1939-1941.

29. Press W, Flannery B, Teukolsky S, Vetterling W. Numerical recipes: the art of scientific computing. Cambridge University Press: New York, 1986.

30. Tam J, Fukumura D, Jain RK. A mathematical model of murine metabolic regulation by leptin: energy balance and defense of a stable body weight. Cell Metabolism 2009;9: 52-63.

31. Ferrannini G, Hach T, Crowe S, Sanghvi A, Hall KD, Ferrannini E. Energy Balance After Sodium Glucose Cotransporter 2 (SGLT2) Inhibition. Diabetes Care 2015.

32. Yi TM, Huang Y, Simon MI, Doyle J. Robust perfect adaptation in bacterial chemotaxis through integral feedback control. Proceedings of the National Academy of Sciences of the United States of America 2000;97: 4649-4653.

33. Heshka S, Anderson JW, Atkinson RL, Greenway FL, Hill JO, Phinney SD, et al. Weight loss with self-help compared with a structured commercial program: a randomized trial. JAMA 2003;289: 1792-1798.

34. Franz MJ, VanWormer JJ, Crain AL, Boucher JL, Histon T, Caplan W, et al. Weight-loss outcomes: a systematic review and meta-analysis of weight-loss clinical trials with a minimum 1-year follow-up. J Am Diet Assoc 2007;107: 1755-1767. 
35. Ravussin E, Redman LM, Rochon J, Das SK, Fontana L, Kraus WE, et al. A twoyear randomized controlled trial of human caloric restriction: feasibility and effects on predictors of health span and longevity. Journal of Gerontology 2015.

36. Maclean PS, Bergouignan A, Cornier MA, Jackman MR. Biology's response to dieting: the impetus for weight regain. Am J Physiol Regul Integr Comp Physiol 2011;301: R581-600.

37. Keys A. The biology of human starvation. University of Minnesota Press: Minneapolis, 1950.

38. Heyman MB, Young VR, Fuss P, Tsay R, Joseph L, Roberts SB. Underfeeding and body weight regulation in normal-weight young men. Am J Physiol 1992;263: R250-257.

39. Roberts SB, Fuss P, Heyman MB, Evans WJ, Tsay R, Rasmussen H, et al. Control of food intake in older men. JAMA 1994;272: 1601-1606.

40. Winkels RM, Jolink-Stoppelenburg A, de Graaf K, Siebelink E, Mars M, de Groot L. Energy intake compensation after 3 weeks of restricted energy intake in young and elderly men. Journal of the American Medical Directors Association 2011;12: 277-286.

41. Blundell JE, Finlayson G, Gibbons C, Caudwell P, Hopkins M. The biology of appetite control: Do resting metabolic rate and fat-free mass drive energy intake? Physiol Behav 2015;152: 473-478.

42. Dulloo AG, Jacquet J, Girardier L. Poststarvation hyperphagia and body fat overshooting in humans: a role for feedback signals from lean and fat tissues. Am J Clin Nutr 1997;65: 717-723.

43. Almiron-Roig E, Palla L, Guest K, Ricchiuti C, Vint N, Jebb SA, et al. Factors that determine energy compensation: a systematic review of preload studies. Nutr Rev 2013;71: 458-473.

44. Bellisle F, Perez C. Low-energy substitutes for sugars and fats in the human diet: impact on nutritional regulation. Neuroscience and biobehavioral reviews 1994;18: 197-205.

45. Stubbs RJ, Harbron CG, Murgatroyd PR, Prentice AM. Covert manipulation of dietary fat and energy density: effect on substrate flux and food intake in men eating ad libitum. Am J Clin Nutr 1995;62: 316-329.

46. Stubbs RJ, Ritz P, Coward WA, Prentice AM. Covert manipulation of the ratio of dietary fat to carbohydrate and energy density: effect on food intake and energy balance in free-living men eating ad libitum. Am J Clin Nutr 1995;62: 330-337. 
47. Lovejoy JC, Smith SR, Bray GA, DeLany JP, Rood JC, Gouvier D, et al. A paradigm of experimentally induced mild hyperthyroidism: effects on nitrogen balance, body composition, and energy expenditure in healthy young men. J Clin Endocrinol Metab 1997;82: 765-770.

48. Sumithran P, Prendergast LA, Delbridge E, Purcell K, Shulkes A, Kriketos A, et al. Long-term persistence of hormonal adaptations to weight loss. $N$ Engl J Med 2011;365: 1597-1604.

49. Speakman JR, Levitsky DA, Allison DB, Bray MS, de Castro JM, Clegg DJ, et al. Set points, settling points and some alternative models: theoretical options to understand how genes and environments combine to regulate body adiposity. Disease models \& mechanisms 2011;4: 733-745.

50. Greenberg I, Stampfer MJ, Schwarzfuchs D, Shai I. Adherence and success in long-term weight loss diets: the dietary intervention randomized controlled trial (DIRECT). J Am Coll Nutr 2009;28: 159-168.

51. Franz MJ. The dilemma of weight loss in diabetes. Diabetes Spectrum 2007;20: 133-136.

52. Devineni D, Curtin CR, Polidori D, Gutierrez MJ, Murphy J, Rusch S, et al. Pharmacokinetics and pharmacodynamics of canagliflozin, a sodium glucose cotransporter 2 inhibitor, in subjects with type 2 diabetes mellitus. Journal of clinical pharmacology 2013;53: 601-610.

53. Sha S, Polidori D, Heise T, Natarajan J, Farrell K, Wang SS, et al. Effect of the sodium glucose co-transporter 2 inhibitor canagliflozin on plasma volume in patients with type 2 diabetes mellitus. Diabetes Obes Metab 2014;16: 1087-1095.

54. Polidori D, Mari A, Ferrannini E. Canagliflozin, a sodium glucose co-transporter 2 inhibitor, improves model-based indices of beta cell function in patients with type 2 diabetes. Diabetologia 2014;57: 891-901.

55. Greenway FL. Physiological adaptations to weight loss and factors favouring weight regain. Int J Obes (Lond) 2015;39: 1188-1196.

56. Rosenbaum M, Hirsch J, Gallagher DA, Leibel RL. Long-term persistence of adaptive thermogenesis in subjects who have maintained a reduced body weight. Am J Clin Nutr 2008;88: 906-912. 


\begin{tabular}{cll}
\hline Parameter & Value & Description \\
\hline$\gamma_{F M}$ & $3.2 \mathrm{kcal} / \mathrm{kg} / \mathrm{d}$ & Energy expenditure rate of fat mass \\
$\gamma_{F F M}$ & $22 \mathrm{kcal} / \mathrm{kg} / \mathrm{d}$ & Energy expenditure rate of fat free mass \\
$\delta_{0}$ & $10 \mathrm{kcal} / \mathrm{kg} / \mathrm{d}$ & Physical activity at baseline \\
$\Delta \delta$ & $0 \mathrm{kcal} / \mathrm{kg} / \mathrm{d}$ & Physical activity changes \\
$\eta_{F M}$ & $180 \mathrm{kcal} / \mathrm{kg}$ & Energy cost of fat turnover \\
$\eta_{F F M}$ & $230 \mathrm{kcal} / \mathrm{kg}$ & Energy cost of protein turnover \\
$\rho_{F M}$ & $9300 \mathrm{kcal} / \mathrm{kg}$ & Energy density of fat mass \\
$\rho_{F F M}$ & $1100 \mathrm{kcal} / \mathrm{kg}$ & Energy density of fat free mass \\
$\beta$ & 0.24 & Dietary and adaptive thermogenesis \\
$U G E$ & $360 \mathrm{kcal} / \mathrm{d}$ & Energy loss due to increased urinary \\
& & glucose excretion with canagliflozin \\
& & (300 mg/d) \\
\hline
\end{tabular}

Table 1. Mathematical model parameters. 


\begin{tabular}{lcc}
\hline Characteristic & $\begin{array}{c}\text { Placebo } \\
(\mathbf{n}=\mathbf{8 9})\end{array}$ & $\begin{array}{c}\text { Canagliflozin } \\
(\mathbf{n}=\mathbf{1 5 3})\end{array}$ \\
\hline Sex, n (\%) & & \\
Male & $47(53)$ & $66(43)$ \\
Female & $42(47)$ & $87(57)$ \\
Age (years) & $57 \pm 10$ & $55 \pm 11$ \\
BW (kg) & $88 \pm 18$ & $87 \pm 21$ \\
BMI (kg/m ${ }^{2}$ ) & $32 \pm 6$ & $32 \pm 6$ \\
Waist circumference (cm) & $106 \pm 13$ & $105 \pm 15$ \\
Race, n (\%) & & \\
White & $62(70)$ & $106(69)$ \\
Black or African American & $2(2)$ & $7(5)$ \\
Asian & $15(17)$ & $24(16)$ \\
Other & $\dagger$ \\
HbA1c (\%) & $10(11)$ & $16(10)$ \\
Fasting plasma glucose (mmol/L) & $7.5 \pm 0.6$ & $7.9 \pm 0.8$ \\
Diabetes duration $(y)$ & $3.2 \pm 2.1$ & $9.3 \pm 2.4$ \\
eGFR (mL/min/1.73m $\left.{ }^{2}\right)$ & $85 \pm 21$ & $3 \pm 4$ \\
\hline
\end{tabular}

Table 2. Baseline characteristics of the study subjects. Data are mean \pm SD unless

otherwise indicated. ${ }^{\dagger}$ Includes American Indian or Alaska Native, Native Hawaiian or other Pacific Islander, multiple, other or not reported 


\section{FIGURE LEGENDS}

Figure 1. Body weight and energy intake changes during placebo and SGLT2 inhibition.

(A) Average body weight measurements in the placebo group ( $\square$ ) and SGLT2 inhibition group (ם) along with mathematical model simulations depicted as red and blue curves, respectively. (B). Calculated energy intake changes in the placebo group $(\diamond)$ and the SGLT2 inhibitor group $(\diamond)$ along with the mathematical model simulations. Mean \pm 95\% CI.

Figure 2. Characterization of feedback control of energy intake in subjects with type 2 diabetes treated with canagliflozin (18). (A) Observed changes in body weight ( $\boldsymbol{\square}$ ) and the simulated changes with proportional control (solid red curve) or integral control (dashed red curve) of energy intake. (B) Calculated changes in energy intake $(\diamond)$ and the simulated changes with or proportional control (solid blue curve) integral control (dashed blue curve) of energy intake. Mean $\pm 95 \%$ CI.

Figure 3. Energy balance dynamics during a lifestyle intervention for weight loss (33). (A) Average body weight ( $\boldsymbol{\square}$ ) typically decreases and reaches a plateau after 6-8 months of a lifestyle intervention followed by slow weight regain. (B) Energy expenditure changes relatively little during the intervention (solid orange curve) whereas energy intake initially drops by a large amount followed by an exponential return towards baseline (solid blue curve). The homeostatic feedback from the body weight loss signals a large increase in homeostatic drive to eat (dashed curve) that is resisted by the attempt to sustain the intervention. (C) The average effort during the intervention was defined as the difference between the homeostatic drive to eat and the actual energy intake. A 
bioRxiv preprint doi: https://doi.org/10.1101/051045; this version posted April 29,2016 . The copyright holder for this preprint (which was not certified by peer review) is the author/funder, who has granted bioRxiv a license to display the preprint in perpetuity. It is made available under aCC-BY-ND 4.0 International license.

substantial effort persists during the intervention despite a return to near baseline energy

intake. Mean \pm 95\% CI. 
A
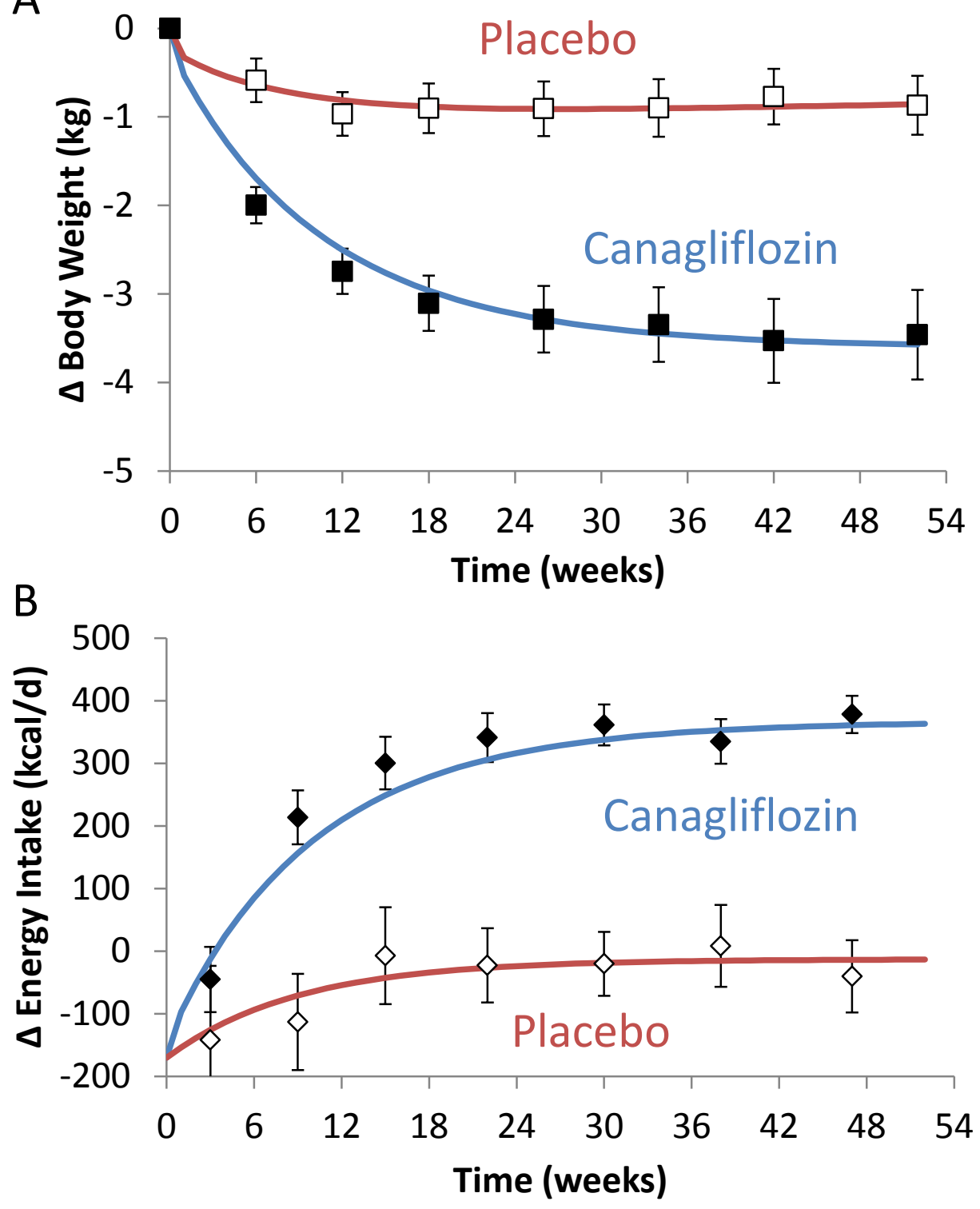

Figure 1 


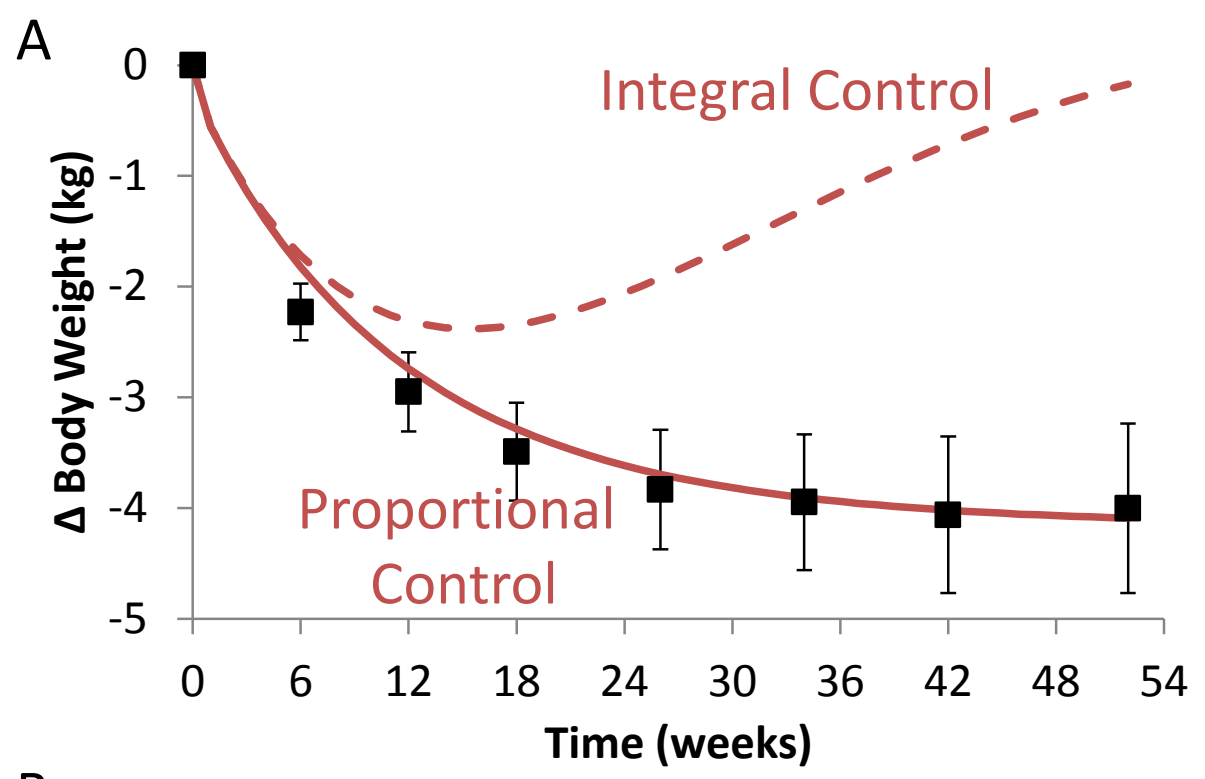

B

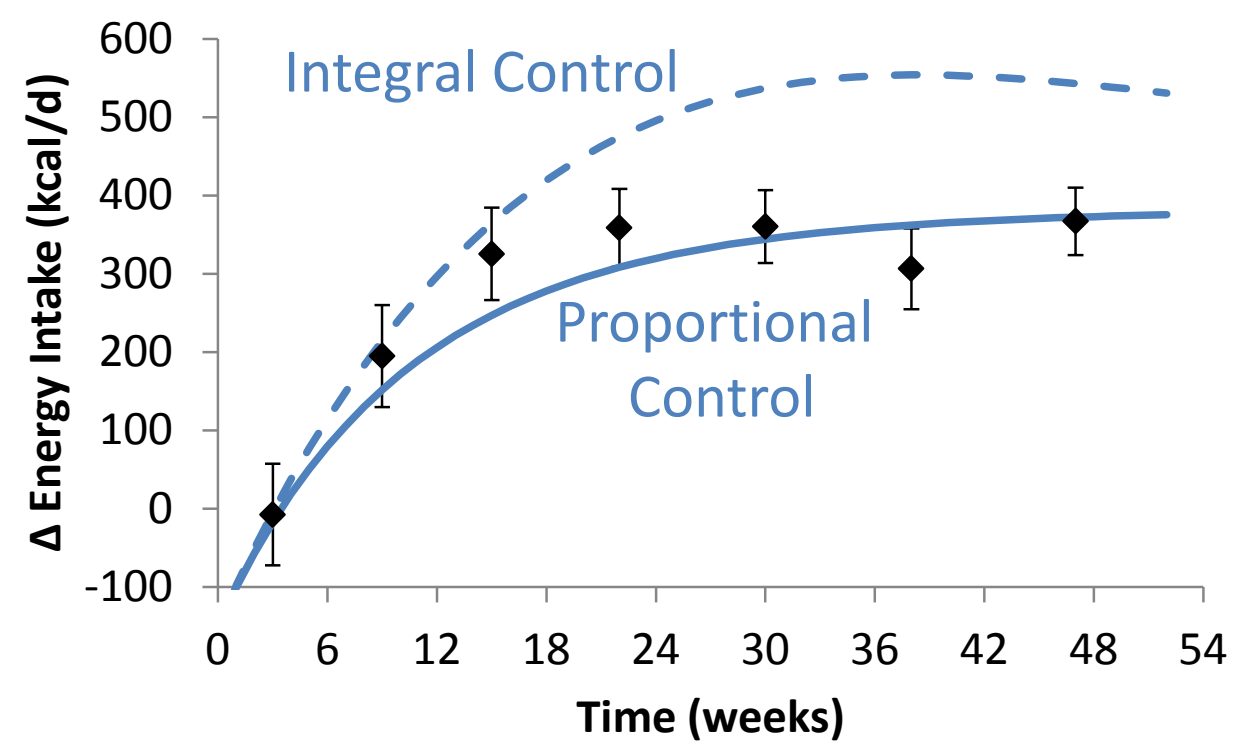

Figure 2 
A
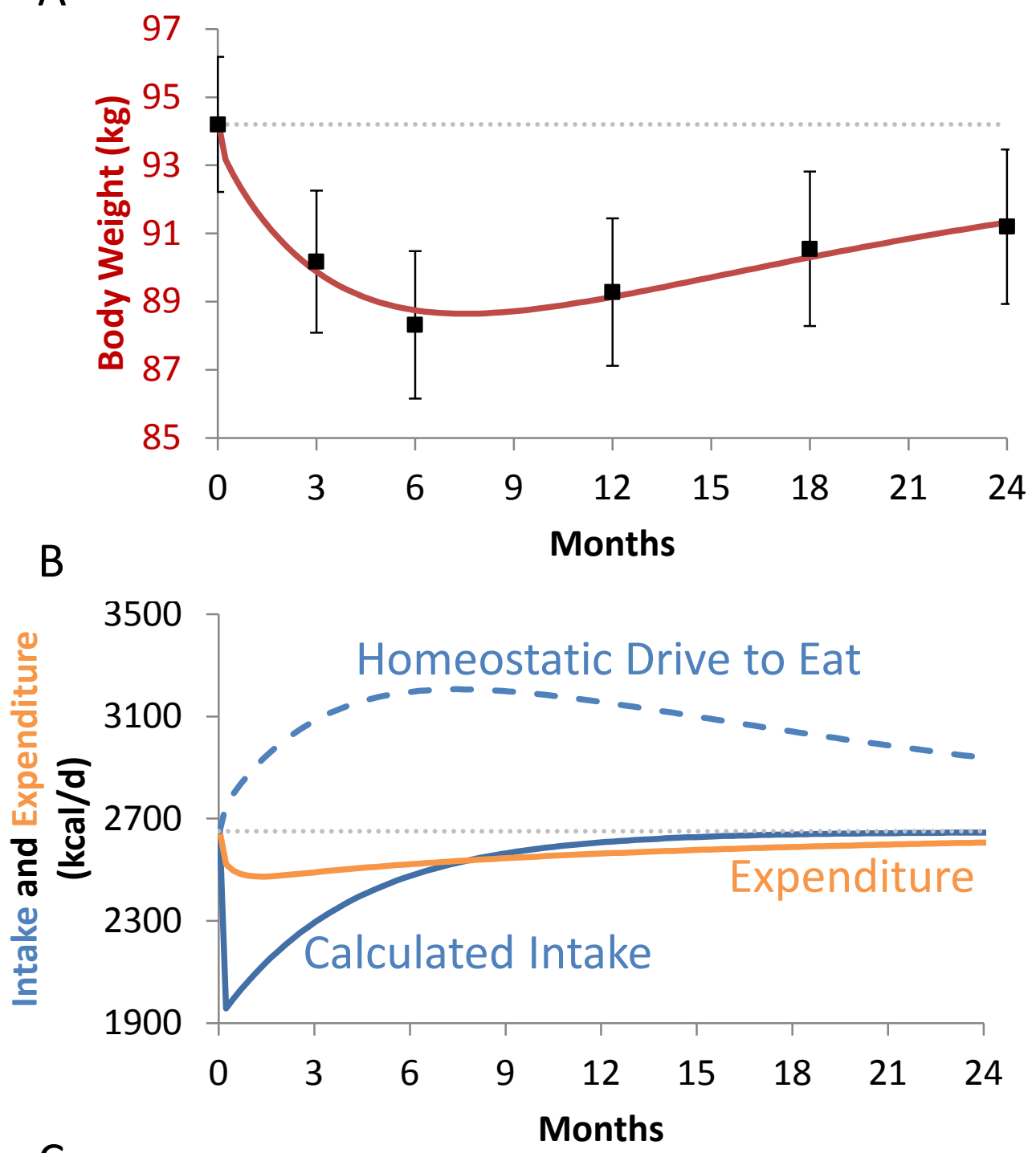

C

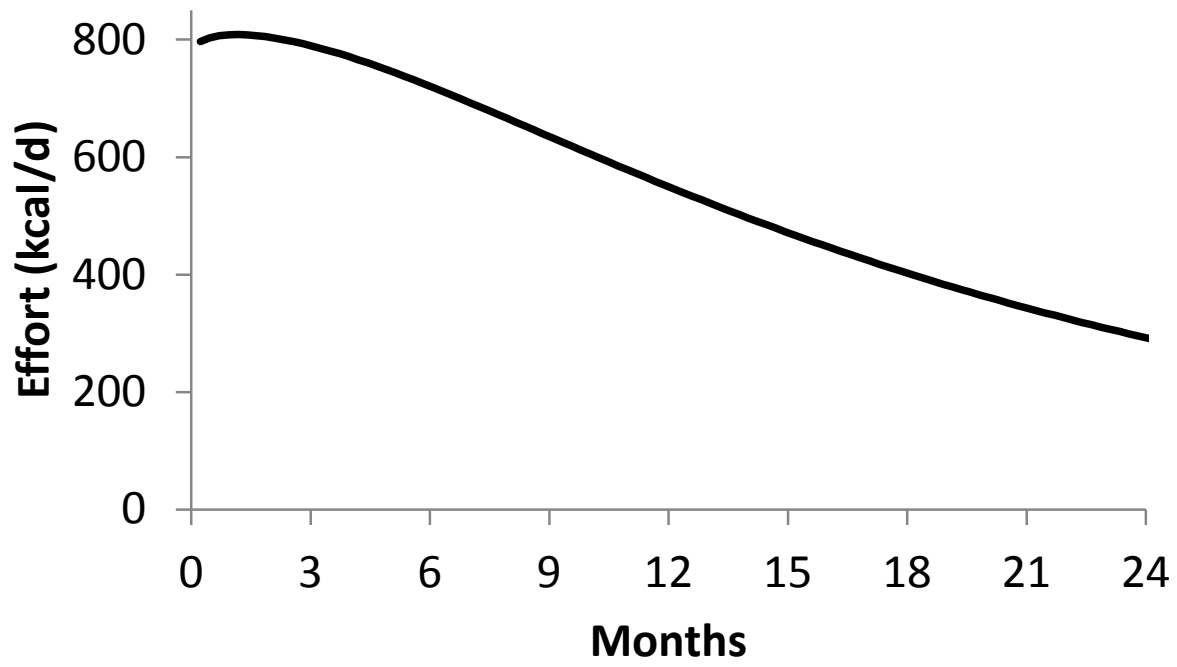

Figure 3 